Rev Chil Salud Pública 2012; Vol 16 (3): $235-240$

\section{Tema de actualidad}

\title{
¿Es la construcción de un hospital antes que nada una promesa populista?
}

\author{
IS PROPOSING THE CONSTRUCTION OF A NEW HOSPITAL ESSENTIALLY A POPULIST \\ PROPOSAL?
}

\section{Introducción}

La Ley de Roemer* establece que cualquier hospital que se instale tendrá máxima ocupación. Los médicos se encargarán de llenarlo y las autoridades mostrarán, entonces, lo necesario que era construirlo. Se refuerza así el rol del hospital en el imaginario colectivo en momentos en que la principal estrategia de atención está basada en el cuidado y seguimiento cercano de los problemas de salud, tarea principal de la atención primaria.

En este artículo se analizarán algunos casos de hospitales prometidos por las autoridades y que, en opinión de este autor, carecían de fundamentos técnicos para su construcción. Se examinarán los casos del Hospital de Quilicura; la reposición del Hospital Félix Bulnes, y el nuevo Hospital de Puente Alto. Se expondrán estudios recientes que utilizando la metodología propuesta por el Ministerio de Salud (Minsal) muestran que en muchos casos existía insuficiente aprovechamiento de la infraestructura existente (optimización de la oferta en el lenguaje Minsal), para satisfacer las legítimas necesidades de acceso a la atención de salud de la población usuaria. Se plantearán también alternativas intermedias de más rápida y fácil implementación, más económicas y con alta aceptación ciudadana, previas a la construcción de un hospital y de las cuales hay buenos ejemplos en la red chilena.

\section{Metodología}

Los antecedentes que se examinarán se han obtenido de los estudios de red asistencial del Servicio de Salud Metropolitano Occidente (SSMOc) $)^{* *}$; del Servicio de Salud Metropolitano Norte (SSMN) ${ }^{* * *}$ y de la red municipal de Puente Alto ${ }^{* * * *}$. Los estudios de red asistencial buscan establecer las necesidades de inversión de un determinado sistema de atención para responder a la demanda de su población usuaria en un horizonte de 10 a 15 años.

En los estudios de red asistencial se utilizó la metodología recomendada por el Minsal ${ }^{*, * *}$ con las siguientes etapas:

- Levantamiento de la capacidad de atención existente en atención primaria, especialidades, hospitalizaciones y capacidad quirúrgica (oferta).

- Estimación de la demanda por atenciones en un horizonte de 10 años. Para ello se recogen las necesidades de salud expresadas por la población a través de grupos focales o encuestas; se analizan proyecciones demográficas y epidemiológicas, complementadas con estudios urbanos y de geografía

\section{ANTONIO INFANTE(1)}

(1)Director de Epidemiología y Gestión Ltda. Av Bustamante 124 Dpto G. Providencia, Santiago. Chile. ainfantebarros@gmail.com
* Roemer Milton, Hospital utilization and the supply of physicians, JAMA, 1961

** Estudio de la Red Asistencial del SSMOc, Epidemiología y Gestión y SSMOc, 2009

*** Estudio de la Red Asistencial del SSMN, Epidemiología y Gestión y SSMN, 2011

**** Flujos de atención y capacidad docente de la red de atención primaria de Puente Alto. Instituto Salud Futuro de la Universidad Andrés Bello y Epidemiología y Gestión, 2011. 
territorial, para con esos antecedentes estimar el volumen futuro de actividades (demanda potencial).

- Comparación entre la oferta existente y la demanda potencial, también llamado estudio de brecha asistencial (brecha).

- Aplicación de estándares de rendimiento médico, de infraestructura y equipamiento a la capacidad existente. Los estándares se generan por consenso de acuerdo a las mejores prácticas nacionales e internacionales (optimización de la oferta).

- Determinación de las áreas críticas y búsqueda de alternativas de solución para responder a la demanda estimada en los próximos 10 años. Entre las alternativas para solucionar las brechas se deben considerar:

- Exigencias al modelo de atención.

- Gestión de listas de espera.

- Inversión en tecnologías.

- Inversión en establecimientos de salud que deben considerar desde centros de salud, SAPU y eventualmente hospitales.

- Localización territorial de la nueva infraestructura: se debe basar en modelos de atracción, semejantes a los que usan las grandes tiendas para instalar sus locales***, que utilizan información sobre localización de la demanda, conectividad y las diversas ofertas sociales para seleccionar la complejidad del nuevo establecimiento teniendo siempre como objetivo responder oportunamente a las necesidades de la población con el menor desplazamiento.

\section{Resultados}

\section{Hospital de Quilicura****}

El estudio de la red mostró inequidades de acceso de las comunas alejadas de los centros hospitalarios del complejo asistencial Norte, tanto en tasas de consulta de especialidades, hospitalizaciones y cirugías. Las listas de espera así lo confirmaban (Tabla 1).
Tabla 1. Inequidades de acceso por comunas. SSMN 2009

\begin{tabular}{lrrrr}
\hline & $\begin{array}{r}\text { Especialidades }^{a} \\
\text { San José }\end{array}$ & $\begin{array}{c}\text { Hospitalizaciones del Río }^{b} \\
\text { San José }\end{array}$ & R. del Río \\
\hline Conchalí & 0,80 & 0,74 & 80 & 120 \\
Huechuraba & 0,50 & 0,53 & 58 & 70 \\
Independencia & 1,39 & 1,36 & 137 & 222 \\
Quilicura & 0,49 & 0,54 & 41 & 57 \\
Recoleta & 0,69 & 0,79 & 77 & 119 \\
Colina & 0,41 & 0,49 & 57 & 68 \\
Lampa & 0,36 & 0,33 & 57 & 82 \\
Tiltil & 0,35 & 0,44 & 48 & 64 \\
\hline
\end{tabular}

${ }^{a}$ Consultas hab/año infantil y adulto

${ }^{b}$ Egresos por mil habitantes infantil o adulto

Fuente: Estudio Red Asistencial SSMN

La inequidad era mayor en el Hospital San José que en el Hospital Roberto del Río. El problema tendía a agravarse porque estas comunas, Quilicura, Lampa y Colina, eran las que experimentaban una mayor tasa de crecimiento poblacional. La opinión de los usuarios confirma esa realidad manifestando críticas a la baja resolutividad de la atención primaria; pocas esperanzas de acceder a consultas de especialistas, procedimientos u hospitalización, y utilizando, en cambio, cada vez con mayor frecuencia, la atención de urgencia o la medicina privada.

\begin{abstract}
"Poder ir a especialista. Tener exámenes. Sí po', uno ¿dónde va primero? Al consultorio, si tienes que ir donde un especialista, pueden ser meses de espera para llegar a un tratamiento...entonces a lo particular, ¿dónde? en cualquier sitio. En cualquier parte en el centro. Por ejemplo, las radiografías, las dos salen como veinte mil pesos, como particular."
\end{abstract}

Fuente: Estudio Red Asistencial SSMN

Al analizar la capacidad de atención de la red (oferta) se encontró que habían disminuido las consultas electivas en atención primaria y aumentado las derivaciones (menor capacidad

\footnotetext{
* Guía metodológica para estudios de preinversión hospitalaria, Minsal 2001.

** Orientaciones para el proceso de diseño de la red asistencial de los servicios de salud, Minsal, octubre 2010.

*** Al aplicar modelos de atracción el hospital se comporta igual que un "mall" y los Sapu con complejidad resultan equivalentes a los "strip centers" que se ubican en esquinas estratégicas de la ciudad, que cuentan con una farmacia, un pequeño supermercado, una lavandería y una sucursal bancaria.

**** La Tercera, 25 de septiembre de 2008.
} 
resolutiva), con un rendimiento médico inferior al $50 \%$ de su capacidad y, por otra parte, había disminuido la capacidad de recepción de consultas nuevas por parte de los especialistas, privilegiando estos últimos un mayor número de controles de sus consultantes: 5.6 controles por cada paciente nuevo en circunstancias que en los hospitales del resto de la Región Metropolitana variaban entre 2.5 y 3 los controles por paciente nuevo. En la Tabla 2 se aprecia el aumento de capacidad de recepción de pacientes nuevos al ajustar el número de controles.

Tabla 2. Optimización de consultas de especialidad Complejo San José (para un total de consultas de 230.000).

\begin{tabular}{lccl}
\hline Escenario & Controles & $\begin{array}{l}\text { Controles por } \\
\text { caso nuevo }\end{array}$ & $\begin{array}{l}\text { Consultas } \\
\text { nuevas }\end{array}$ \\
\hline Actual 2010 & 195.362 & 5.6 & 34.638 \\
1 & 188.182 & 4.5 & 41.818 \\
2 & 178.889 & 3.5 & 51.111 \\
3 (óptimo) & 164.286 & 2.5 & 65.714 \\
\hline
\end{tabular}

Fuente: Estudio Red Asistencial SSMN

También en la gestión de camas el hospital San José mostraba promedios de estada sensiblemente más altos que los mejores centros públicos y privados de Santiago (Tabla 3).

Tabla 3. Comparación de tiempos de estada con mejores indicadores nacionales.

\begin{tabular}{lcc}
\hline & Prom Días & $\begin{array}{c}\text { \% de egresos }<10 \text { años y } \\
\text { embarazadas }\end{array}$ \\
\hline CHSJ & 6,6 & 41,7 \\
Alemana & 3,6 & 28 \\
Católica & 4,4 & 21,3 \\
J.J. Aguirre & 5,3 & 18,2 \\
Padre H & 4,2 & 55 \\
Sótero & 5,3 & 35,2 \\
\hline
\end{tabular}

Fuente: DEIS Minsal
Se aprecia que el Hospital San José tiene mayores tiempos de estada con una alta proporción de egresos obstétricos de corta estadía (3.1 días), lo que indica una estadía prolongada en los otros servicios (una buena experiencia internacional como el Kaiser Permanente en USA tiene 3.9 días). Otro dato interesante en el análisis de la oferta fue que los ingresos a hospitalización se producían en su mayoría a través de los servicios de urgencia y que un número importante de ellos, $60 \%$ en el Roberto del Río y $33 \%$ en el San José, estaba entre uno o dos días, principalmente para descartar sospechas diagnósticas.

Al optimizar la oferta mejorando la capacidad resolutiva de los especialistas e incorporando estándares más exigentes a la gestión clínica hospitalaria con mayor énfasis en la ambulatorización de actividades, se encontró que el Complejo San José podía absorber la demanda al 2020 , incluso haciéndose cargo de la atención de cánceres, hoy en el Instituto del Cáncer, que pasaría a ser un verdadero centro de referencia nacional y no un servicio clínico especializado del SSMN, y también de las derivaciones que se hacen al J.J. Aguirre que consumen parte del presupuesto del SSMN. Las optimizaciones del Hospital Roberto del Río y del Instituto Psiquiátrico arrojaron holguras en recursos humanos y alternativas de gestión clínica que permitirían reforzar la atención primaria y aumentar la capacidad de hospitalización absorbiendo listas de espera.

La optimización de la oferta no aseguraba una mejoría en la equidad de acceso para las comunas distantes y de crecimiento rápido del complejo hospitalario Norte. Sin embargo, por otra parte no se justificaba la instalación de otro hospital dado que los existentes eran capaces de absorber la demanda hasta el 2020. Había que buscar soluciones alternativas.

Las alternativas propuestas para el problema de la equidad enfatizaron la mayor resolutividad de la APS municipal, siguiendo como ejemplo la experiencia del Instituto Psiquiátrico que ha- 
bía destinado horas de becados de los últimos años y un arsenal farmacológico más completo a centros municipales (Cosam), descongestionando su centro de especialidades y mejorando la cobertura en poblaciones que no accedían, como los adolescentes. Sin embargo, no se solucionaba el acceso oportuno a especialidades y tecnología. Para ello y usando el modelo de atracción, se planteó la posibilidad de transformar los Sapu de Quilicura y Colina en lo que se denominó Sapu Plus u Hospital Comunitario*. Se trata de un establecimiento dotado de imagenología (ecógrafo y Rx osteopulmonar), laboratorio básico (kits de cintas), camas de observación y urgencia dental, capaz de resolver las sospechas diagnósticas que se hospitalizaban en el Complejo Norte y que contará, además, con pabellón y salas de procedimiento para que durante el día llegaran especialistas para atender problemas de fácil resolución ambulatoria. Además de Quilicura y Colina, el modelo proponía uno en Recoleta Norte para mejorar el acceso de Huechuraba y los extremos norte de Conchalí y Recoleta.

\section{Hospital Félix Bulnes}

El caso de este hospital es diferente ya que se trata de una reposición después de su colapso en el terremoto del 27F. Sin embargo, las decisiones al respecto podían basarse en un estudio de red asistencial realizado en 2009 con horizonte 2022.

Las proyecciones demográficas y urbanísticas para el sector occidente señalaban que la población usuaria se mantendría constante, con un área de crecimiento futuro usuaria del sistema público en Pudahuel Sur y también expansión urbana en el sector Talagante-Peñaflor, pero de sectores más acomodados. El área jurisdiccional del Félix Bulnes (Cerro Navia, Quinta Normal, Renca) experimentaría una importante reducción de población principalmente joven y, por lo tanto, la demanda futura disminuiría.
Tabla 4. Variación de poblaciones usuarias de hospitales al año 2022, Servicio de Salud Metropolitano Occidente.

\begin{tabular}{|c|c|c|c|}
\hline Hospital & Comunas & Personas & \% Variación \\
\hline $\begin{array}{l}\text { Félix } \\
\text { Bulnes }\end{array}$ & $\begin{array}{l}\text { Renca, } \\
\text { Cerro Navia } \\
\text { Quinta } \\
\text { Normal }\end{array}$ & -67.000 & $-19 \%$ \\
\hline $\begin{array}{l}\text { San Juan } \\
\text { de Dios }\end{array}$ & $\begin{array}{l}\text { Lo Prado y } \\
\text { Pudahuel }\end{array}$ & +44.000 & $+12 \%$ \\
\hline Talagante & $\begin{array}{l}\text { Provincia } \\
\text { Talagante }\end{array}$ & +43.000 & $+16 \%$ \\
\hline Melipilla & $\begin{array}{l}\text { Provincia } \\
\text { Melipilla }\end{array}$ & +20.800 & $+8 \%$ \\
\hline
\end{tabular}

Fuente: Estudio Red Asistencial SSMOc Melipilla

Los balances de recursos humanos del Servicio Occidente mostraban un desajuste respecto a la demanda, con importantes listas de espera en especialidades falentes como otorrino, oftalmología, traumatología infantil, urología y dermatología y, sin embargo, un importante excedente de capacidad en medicina, pediatría $y$, en algunos lugares, ginecobstetricia. Infraestructura habilitada en los últimos años como el CRS Salvador Allende mostraban una ocupación inferior al 50\%, algo que probablemente cambiaría con la ampliación de la Línea 5 del Metro y el crecimiento de Pudahuel Sur.

Las recomendaciones se centraron en mejorar la capacidad resolutiva del nivel primario, con fortalecimiento de los Sapu del área de Pudahuel y Renca (el río Mapocho es una importante barrera de acceso), instalándoles camas de observación. Se propuso también una importante ampliación de capacidad del Hospital de Talagante dotándolo de más camas, pabellones y especialistas. Para el Hospital Félix Bulnes se estimaban suficientes 155 camas al final del período 2022.

Sin embargo, respecto al Félix Bulnes las recomendaciones del estudio aparentemente no han sido consideradas ya que se estaría diseñando un hospital de 550 camas, ubicado en

\footnotetext{
* La experiencia más exitosa en este sentido es la evolución de la posta de Maipú que partió adosada a un consultorio para luego instalarse en un centro de especialidades lo que dio paso finalmente a un hospital. También está la experiencia del Hospital de Alto Hospicio que se inició como Sapu para agregársele posteriormente camas de observación.
} 
Cerro Navia*. El modelo de atracción no aconsejaba establecimientos en esa comuna ya que la expansión territorial del servicio occidente se producía hacia Pudahuel Sur, tampoco existían facilidades de desplazamiento de la población comparables con la Línea 5 del Metro y, por último, su área de influencia es cercenada por el río Mapocho. Tampoco el número de camas resiste análisis respecto al crecimiento de la demanda.

Otro de las curiosidades que arrojó el estudio fue la relativa al futuro Hospital de Maipú. Diseñado por el Servicio de Salud Metropolitano Central, no tomó en consideración que la futura estación de transferencia del transporte público de Melipilla, Talagante y Padre Hurtado se ubicaría en la Plaza de Maipú, a metros del nuevo hospital. No se incluyó en el análisis porque eran usuarios del Servicio de Salud Occidente (¿?). Si Maipú hiciera de centro de referencia de esas provincias del sector poniente, el Servicio Occidente podría hacerse cargo de Estación Central y Santiago permitiendo que el Hospital San Borja Arriarán asumiera otras tareas en la región metropolitana.

\section{Nuevo Hospital de Puente Alto}

El crecimiento acelerado de Puente Alto y la saturación del Complejo Sótero del Río explican la demanda histórica de un hospital para esa comuna y así se anunció hace algunos meses. Junto con la próxima inauguración del Hospital de La Florida se iniciará la reposición del Complejo Sótero del Río y la construcción del nuevo Hospital de Puente Alto**.

¿Qué dicen los números? Puente Alto tenía en 201064 egresos por mil habitantes en el Sótero del Río a los que habría que sumar pequeñas cantidades de pacientes hospitalizados en San José de Maipo y en el Hospital Metropolitano de Providencia. La inauguración del Hospital de La Florida le restará al Sótero del Río, el 40\% de su población usuaria, lo que permite proyectar una tasa de egresos futura para Puente Alto de 90 por mil habitantes, cifra superior a las me- jores recomendaciones internacionales de 75 a 80 egresos por mil habitantes ${ }^{* * *}$ y que hace, al menos, dudosa la recomendación de un nuevo hospital para la comuna. Lo que sí es urgente es buscar soluciones para descongestionar el Sótero del Río antes de su reposición y la puesta en marcha del nuevo hospital de La Florida.

Una alternativa que se podría ensayar para descomprimirlo es dotar de camas de observación, la imagenología y el laboratorio ya los tiene, al Sapu del Consultorio Alejandro del Río, un centro tradicional de la comuna y que en sus tiempos fue hospital.

\section{Conclusiones y comentarios}

En este trabajo se han mostrado 3 experiencias relacionadas con promesas de instalación de nuevos hospitales hechas por autoridades del Ministerio de Salud que no responden a los criterios técnicos recomendados por esa misma repartición. En los tres casos las autoridades recogen demandas históricas de las comunidades y hacen anuncios en momentos oportunos políticamente, sin considerar la optimización de la infraestructura existente y tampoco la existencia de otras inversiones que repercutirían favorablemente en la realidad asistencial de esas comunidades.

En los dos estudios de red asistencial quedó en evidencia que una parte de la infraestructura hospitalaria está insuficientemente aprovechada, que la estructura de la dotación médica no se ajusta a las necesidades de la demanda y que su rendimiento puede mejorar. Optimizar el modelo de atención, desarrollando una atención primaria que responda a las expectativas y necesidades de los usuarios aparece como la alternativa más lógica. Sin embargo, el desprestigio actual de la atención municipal, no por su infraestructura sino por su baja resolutividad, demandará esfuerzos que conjuguen inversiones, voluntad política y eventualmente cambios institucionales en su administración. Otra tarea imprescindible para fortalecer el sistema públi-

* $\quad$ El Mercurio, 5 de enero de 2011.

** La Tercera, 31 de julio de 2011.

*** A retrospective analysis of health systems in Denmark and Kaiser Permanente. Anne Frølich, Michaela L Schiøtz,Martin StrandbergLarsen,John Hsu, Allan Krasnik, Finn Diderichsen, Jim Bellows, Jes Søgaard, and Karen White. BMC Health Serv Res. $2008 ; 8: 252$. 
co será superar el desapego con su quehacer asistencial que muestran algunos funcionarios $y$, principalmente, sectores del estamento médico.

Para una mejor utilización de los recursos públicos, además de hacer las correcciones sugeridas en el párrafo anterior, se pueden ensayar modalidades de atención probadas exitosamente en el sistema de salud chileno como in- corporar complejidad en la urgencia de atención primaria o dotar con consejería de especialistas a los centros municipales. En este trabajo se propone, además, la instalación de hospitales comunitarios urbanos (Sapu Plus) como una manera de acercar la resolutividad, la tecnología y las especialidades a la atención primaria, evitando listas de espera y fortaleciendo la capacidad de los centros de salud. 